\section{Cureus}

Received 06/28/2017

Review began 08/23/2017

Review ended 09/17/2017

Published 09/25/2017

\section{C) Copyright 2017}

Batnyam et al. This is an open access article distributed under the terms of the Creative Commons Attribution License CC-BY 3.0., which permits unrestricted use, distribution, and reproduction in any medium, provided the original author and source are credited.

\title{
Hypoglycemia-associated In-stent Thrombosis: Are We Doing Too Much?
}

\author{
Uyanga Batnyam $^{1}$, Nway Ko Ko ${ }^{2}$, Aamir Javaid ${ }^{2}$ \\ 1. Internal Medicine Residency Program, University of Central Florida College of Medicine 2. Internal \\ Medicine, University of Central Florida College of Medicine
}

$\square$ Corresponding author: Uyanga Batnyam, uyanga.batnyam@ucf.edu

Disclosures can be found in Additional Information at the end of the article

\section{Abstract}

Diabetes mellitus is one of the most common modifiable risk factors for coronary artery disease, and its prevalence is rising globally. Persistent hyperglycemia has a well-established cardiovascular risk, and its treatment plays an important role in the prevention of future cardiovascular events. While we improved microvascular complications such as retinopathy, nephropathy, and neuropathy by stringent blood glucose control, the cardiovascular morbidity and mortality in diabetics remain high. Hypoglycemia, on the other hand, is an important side effect of pharmaceutical blood glucose control, especially those who are treated with insulin. Here, we report the case of a 38-year-old man with type 1 diabetes presenting twice with acute ST-elevation myocardial infarction, both in the setting of documented hypoglycemia. There are reported cases of acute cardiovascular events or silent myocardial ischemia associated with hypoglycemia, and we wish to raise awareness for clinicians who treat this special population of patients.

Categories: Cardiology, Internal Medicine, Diabetes \& Endocrinology

Keywords: in-stent thrombosis, diabetes, myocardial infaction, hypoglycemia

\section{Introduction}

The prevalence of diabetes mellitus (DM) is continuously rising; approximately 30.3 million adults in the United States have been diagnosed with DM [1]. Persistent hyperglycemia is a known cardiovascular risk factor, and by tight blood glucose control, improvement in microvascular complications such as retinopathy, nephropathy, and neuropathy has been demonstrated. However, the cardiovascular morbidity and mortality in diabetics remain high despite tight blood glucose control. Hypoglycemia is not an uncommon presentation among patients presenting with acute coronary syndrome (ACS), and we are unaware if that is the cause or the consequence. Multiple large studies have demonstrated poor cardiovascular outcomes with intensive blood glucose control, and there have been reported associations between hypoglycemia, myocardial ischemia, and ACS [2-3].

\section{Case Presentation}

A 38-year-old male with type 1 diabetes mellitus presented to our institute with severe substernal chest pain that started shortly after regaining consciousness from a short duration of hypoglycemic coma with documented blood glucose of $40 \mathrm{mg} / \mathrm{dL}$. The patient had active chest pain upon presentation, and the electrocardiogram showed ST segment (isoelectric section of the electrocardiogram between the end of the $\mathrm{S}$ wave and the beginning of the $\mathrm{T}$ wave) elevations in the inferior leads with reciprocal changes (Figure 1). An emergent left heart catheterization revealed an in-stent thrombosis in his previously placed nine-month-old stent 


\section{Cureus}

despite being strictly compliant with a dual antiplatelet therapy including aspirin and ticagrelor (Figure 2). Of interest, his first ST-elevation myocardial infarction nine months ago also was preceded by a hypoglycemic episode with documented low blood sugar levels, and he subsequently had a drug-eluting stent (DES) implanted in the right coronary artery. We had performed mechanical thrombus aspiration, and placed another DES distal to the previous stent (Figure 3).

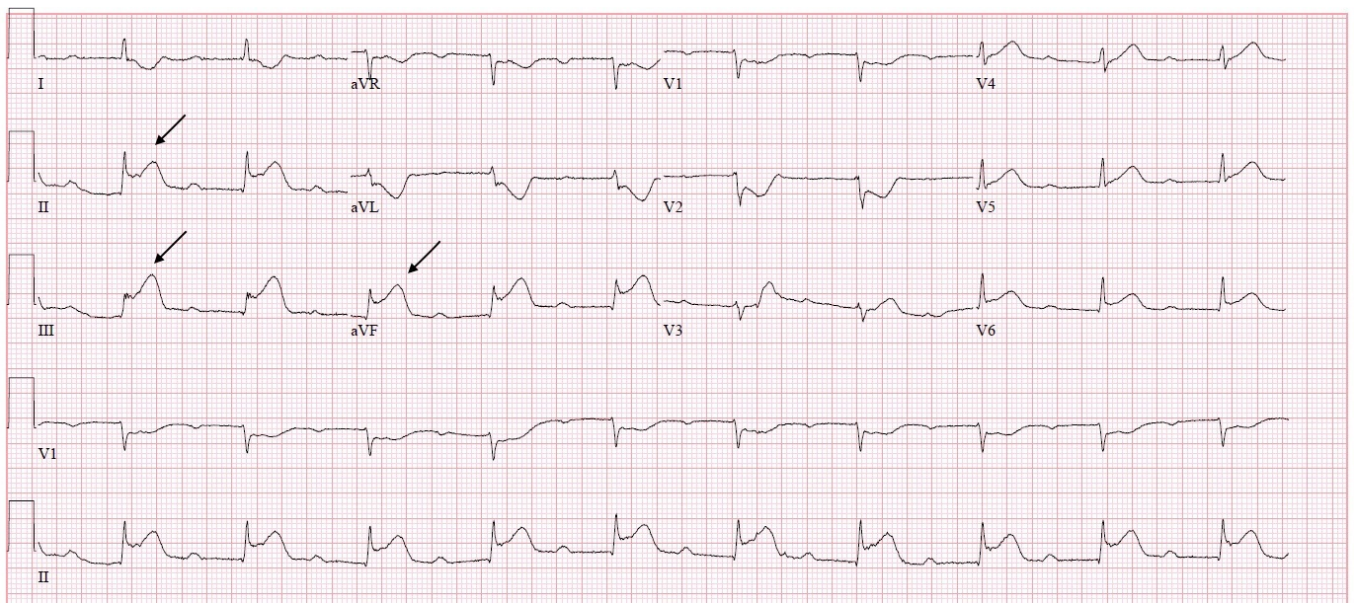

FIGURE 1: Electrocardiogram upon presentation

ST elevation (see arrows) in inferior leads (II, III and aVF) with reciprocal changes. 


\section{Cureus}

\section{FIGURE 2: Total occlusion of right coronary artery}

An emergent left heart catheterization revealed total occlusion of the right coronary artery in the previously placed stent (arrow). There was no flow distal to the occlusion. 


\section{Cureus}

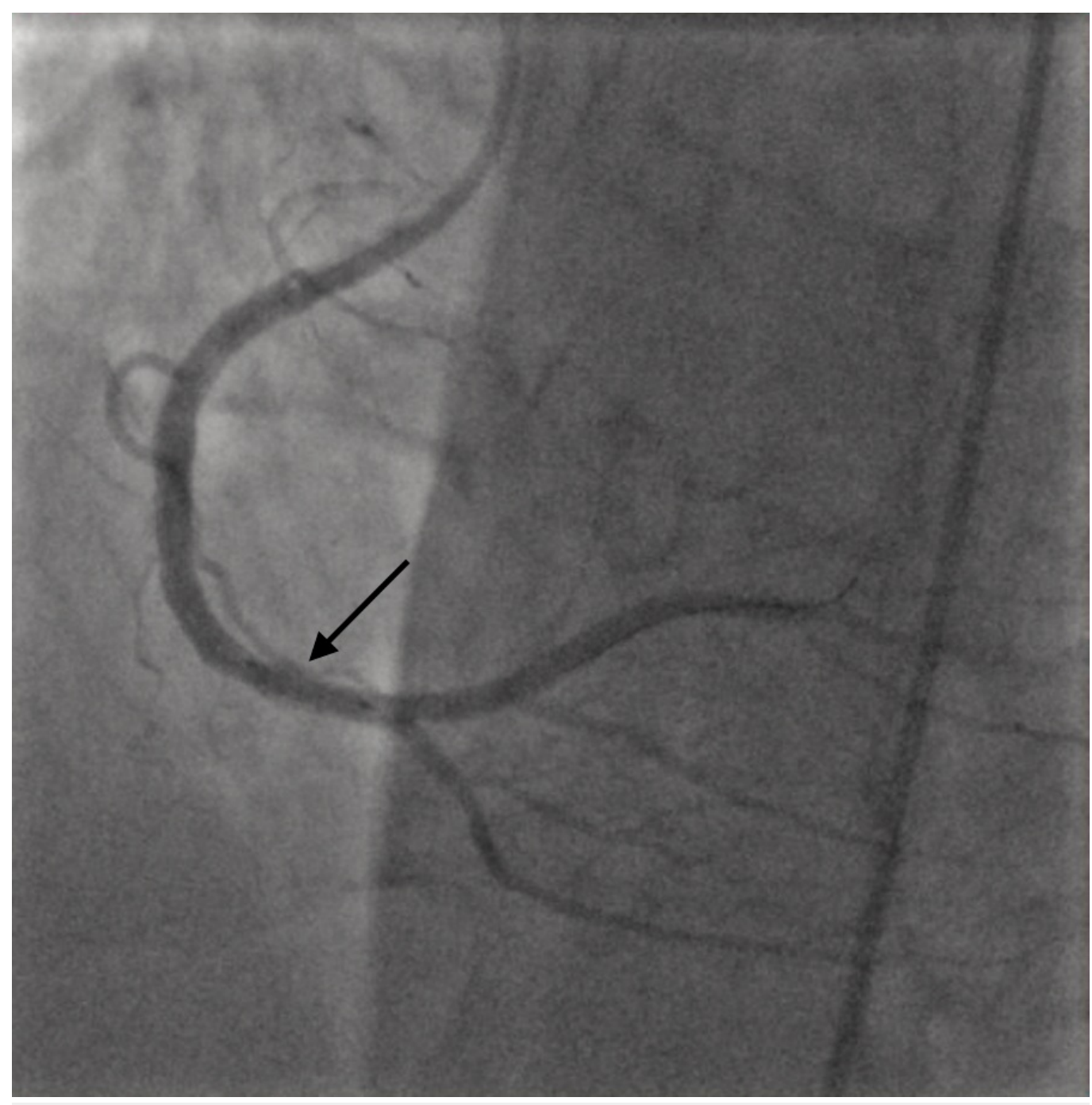

\section{FIGURE 3: Right coronary artery, post-stent placement}

Right coronary artery with TIMI-3 flow (arrow) after mechanical thrombus aspiration and placement of another stent distal to the previously placed stent.

\section{Discussion}

The relationship between hypoglycemia and poor cardiovascular outcomes is complex. Hypoglycemia activates the sympathoadrenal system, causes glucagon and catecholamine surges, thereby causing increased cardiac work and myocardial oxygen demand. Hypoglycemia has been shown to induce inflammatory cascades, and has been associated with enhanced platelet aggregation which is known to promote thrombosis [4-5]. Case reports and early animal models have hypothesized the association between hypoglycemia and ACS, and multiple studies have revealed a documented association between silent myocardial ischemia on a cardiac monitor during asymptomatic hypoglycemic episodes that is recorded by a continuous glucose monitoring in diabetics [6]. There are multiple case reports of ACS in the setting of acute insulin poisoning, particularly in younger adults without established cardiovascular risk factors [7]. Hypoglycemia induced by oral hypoglycemic agents have also been reported to be associated with ACS [8-9]. It is possible that in some cases ACS can be the consequence of hypoglycemia, and the "dead in bed Syndrome" in diabetics could be associated with hypoglycemia-induced adverse cardiovascular events. Physicians might be doing more harm by intense blood glucose control, and large studies are needed to evaluate this potential 
association.

\section{Conclusions}

To the best of our knowledge, we report the first case of hypoglycemia associated stent thrombosis in a patient with type 1 diabetes mellitus. We are proposing special awareness for hypoglycemia in patients with diabetes who are medically treated.

\section{Additional Information \\ Disclosures}

Human subjects: Consent was obtained by all participants in this study. Conflicts of interest: In compliance with the ICMJE uniform disclosure form, all authors declare the following: Payment/services info: All authors have declared that no financial support was received from any organization for the submitted work. Financial relationships: All authors have declared that they have no financial relationships at present or within the previous three years with any organizations that might have an interest in the submitted work. Other relationships: All authors have declared that there are no other relationships or activities that could appear to have influenced the submitted work.

\section{References}

1. National diabetes statistics report, 2017. (2017). Accessed: September 21, 2017: http://www.diabetes.org/assets/pdfs/basics/cdc-statistics-report-2017.pdf.

2. ADVANCE Collaborative Group, Patel A, MacMahon S, et al.: Intensive blood glucose control and vascular outcomes in patients with type 2 diabetes. N Engl J Med. 2008, 358:2560-2572. 10.1056/NEJMoa0802987

3. The Action to Control Cardiovascular Risk in Diabetes Study Group: Effects of intensive glucose lowering in type 2 diabetes. N Engl J Med. 2008, 358:2545-2559. 10.1056/NEJMoa0802743

4. Hanefeld M, Duetting E, Bramlage P: Cardiac implications of hypoglycaemia in patients with diabetes - a systematic review. Cardiovasc Diabetol. 2013, 12:135. 10.1186/1475-2840-12-135

5. Rana OA, Byrne CD, Greaves K: Intensive glucose control and hypoglycaemia: a new cardiovascular risk factor?. Heart. 2014, 100:21-27. 10.1136/heartjnl-2013-303871

6. Zhang JW, Zhou YJ: Association of silent hypoglycemia with cardiac events in non-diabetic subjects with acute myocardial infarction undergoing primary percutaneous coronary interventions. BMC Cardiovasc Disord. 2016, 16:75. 10.1186/s12872-016-0245-Z

7. Kamijo Y, Soma K, Aoyama N, et al.: Myocardial infarction with acute insulin poisoning--a case report. Angiology. 2000, 51:689-693.

8. Chang JH, Tseng CF, Wang JY: Hypoglycemia-induced myocardial infarction: an unusual adverse effect of sulfonylureas. Int J Cardiol. 2007, 115:414-416. 10.1016/j.ijcard.2006.01.062

9. Corley BT, Davenport C, Delaney L, et al.: Hypoglycaemia-induced myocardial infarction as a result of sulphonylurea misuse. Diabet Med. 2011, 28:876-879. 10.1111/j.1464-

5491.2010.03226.x 